\title{
Diseño de una base de datos para la entrevista inicial de tutoría
}

\section{Designing a database to the initial tutoring interview}

\author{
HERNÁNDEZ-TAPIA, Zaila†*, REYNA-ÁNGELES, Omar, AVALOS-MARTÍNEZ, Giovanni y \\ GÓMEZ-MENDOZA, Pablo Hernán
}

Universidad Politécnica De Francisco I. Madero

ID $1^{\mathrm{er}}$ Autor: Zaila, Hernández-Tapia / ORC ID: 0000-0003-2564-3451, Researcher ID Thomson: G-6592-2018, CVU CONACYT ID: 622127

ID $1^{\text {er }}$ Coautor: Omar, Reyna-Ángeles / ORC ID: 0000-0001-6604-9059, Researcher ID Thomson: I-3308-2018, CVU CONACYT ID: 097627

ID $2^{\text {do }}$ Coautor: Giovanni, Avalos-Martínez / ORC ID: 0000-0002-6401-9160

ID $3^{\text {er }}$ Coautor: Pablo Hernán, Gómez-Mendoza / ORC ID: 0000-0002-5635-6837

DOI: $10.35429 / J T A E .2020 .12 .4 .10 .14$

Recibido: 30 de Junio, 2020; Aceptado 20 Octubre, 2020

\begin{abstract}
Resumen
El presente trabajo consiste en el diseño de una base de datos, que permitirá almacenar información obtenida de la entrevista inicial de tutoría, aplicada a los alumnos de nuevo ingreso, la cual se divide en 5 secciones: Datos generales, Información socioeconómica, Antecedentes académicos, Salud y Psicología, el propósito es contar con una herramienta para aplicar la entrevista de forma digital, y optimizar tiempos y materiales requeridos, ya que actualmente se realiza de forma manual lo que genera gastos de impresión y fotocopias que después se convierten en desechos, además de que el procesamiento de la información es complejo. Se utilizará la metodología en cascada ya que se caracteriza por dividir los procesos de desarrollo en sucesivas fases de proyecto y cada una de las fases sirve como punto de partida para la siguiente. Como primera etapa solo se implementará en la carrera de Ingeniería en Sistemas Computacionales y posteriormente se compartirá con los demás programas educativos de la UPFIM.
\end{abstract}

Base de datos, Tutoría, Entrevista

\begin{abstract}
The present work consists of the design of a database, which will allow the storage of information obtained from the initial tutoring interview, applied to new students, which is divided into 5 sections: General Data, Socioeconomic Information, Academic Background, Health and Psychology, the purpose is to have a tool to apply the interview digitally, and optimize time and materials required, since currently it is done manually which generates printing and photocopying costs that later become waste, in addition to the fact that processing the information is complex. The cascade methodology will be used since it is characterized by dividing the development processes in successive project phases and each one of the phases serves as a starting point for the next one. As a first stage, it will only be implemented in the Computer Systems Engineering career and later on it will be shared with the other educational programs of the UPFIM.
\end{abstract}

Database, Tutoring, Interview

Citación: HERNÁNDEZ-TAPIA, Zaila, REYNA-ÁNGELES, Omar, AVALOS-MARTÍNEZ, Giovanni y GÓMEZMENDOZA, Pablo Hernán. Diseño de una base de datos para la entrevista inicial de tutoría. Revista de Tecnología y Educación. 2020. 4-12: 10.14

\footnotetext{
* Correspondencia del Autor (zhernandez@upfim.edu.mx)

$\dagger$ Investigador contribuyendo como primer autor.
} 


\section{Introducción}

En la Universidad Politécnica de Francisco I. Madero se visualiza a las tutorías como una herramienta integrada por áreas de apoyo especializadas, que permiten brindar atención al alumno en su proceso de formación integral. Al ingresar a la universidad y durante su estancia los alumnos tienen asignado a un tutor el cual identifica posibles problemas de índole personal, académico o profesional y en función de ello puede ofrecer alternativas de solución mediante la canalización a las áreas de apoyo especializadas "Psicología, Servicio Médico, Becas, Asesoría".

En este trabajo sólo se presentará la sección de registro de datos generales del alumno. Estos datos se obtienen de 3 documentos oficiales: CURP, Acta de nacimiento y Certificado de Media Superior, lo que permite que la información sea correcta, precisa y validada.

El artículo está organizado de la siguiente manera: En la sección 2 se encuentra la descripción del problema y metodología aplicada, en la sección 3 se presentan las etapas realizadas para lograr el diseño de la base de datos, así como la interfaz de un formulario y finalmente en la sección 4 resultados y conclusiones.

\section{Descripción del problema}

La universidad no cuenta con una herramienta que permita realizar de forma digital la entrevista inicial de tutoría a los alumnos de nuevo ingreso, por lo que el recabar la información, así como su posterior procesamiento involucra mucho tiempo de dedicación y en algunos casos al no contar con ella a tiempo impacta en el \% de canalización del alumno a las áreas de apoyo especializadas de forma oportuna, esto se ve reflejado en los resultados al medir los indicadores de deserción, aprovechamiento, eficiencia terminal e incluso rezago.

\section{Metodología}

Para el desarrollo de este trabajo se aplica la metodología en cascada. Consta de 5 etapas, mostradas en la Figura 1, que se ejecutan una tras otra.

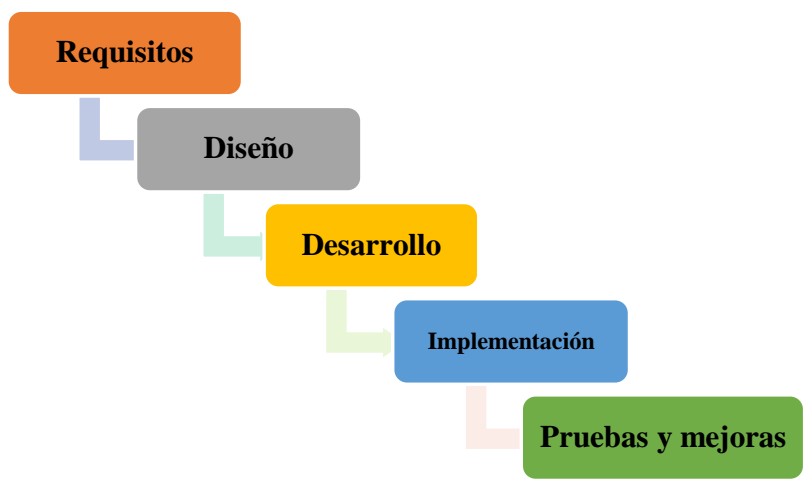

Figura 1: Metodología en cascada

Fuente: Elaboración Propia

Requisitos. En esta etapa se analizan las necesidades de los usuarios finales.

Diseño. Se obtiene y modelo la información que será utilizada para la base de datos y presentada al usuario a través de una interface.

Desarrollo. Se estructura la base de datos en un SMBD (Sistema Manejador de Base de Datos), y se realiza la conexión con las interfaces.

Implementación. En esta etapa el formulario deberá ser utilizado para que se determine la aprobación o su caso modificación a través del usuario final.

Pruebas y mejoras. Se inicia solo en el caso de que exista algún error, modificaciones o mejoras que el usuario final solicite para que esté totalmente satisfecho con los resultados.

\section{Etapas realizadas para lograr el diseño de la base de datos}

1. Requisitos A través de un análisis de requerimientos el cliente determina los datos que se deben almacenar en la base de datos que estará conectada al formulario. Por lo que proporciona la entrevista impresa que se aplica actualmente. 


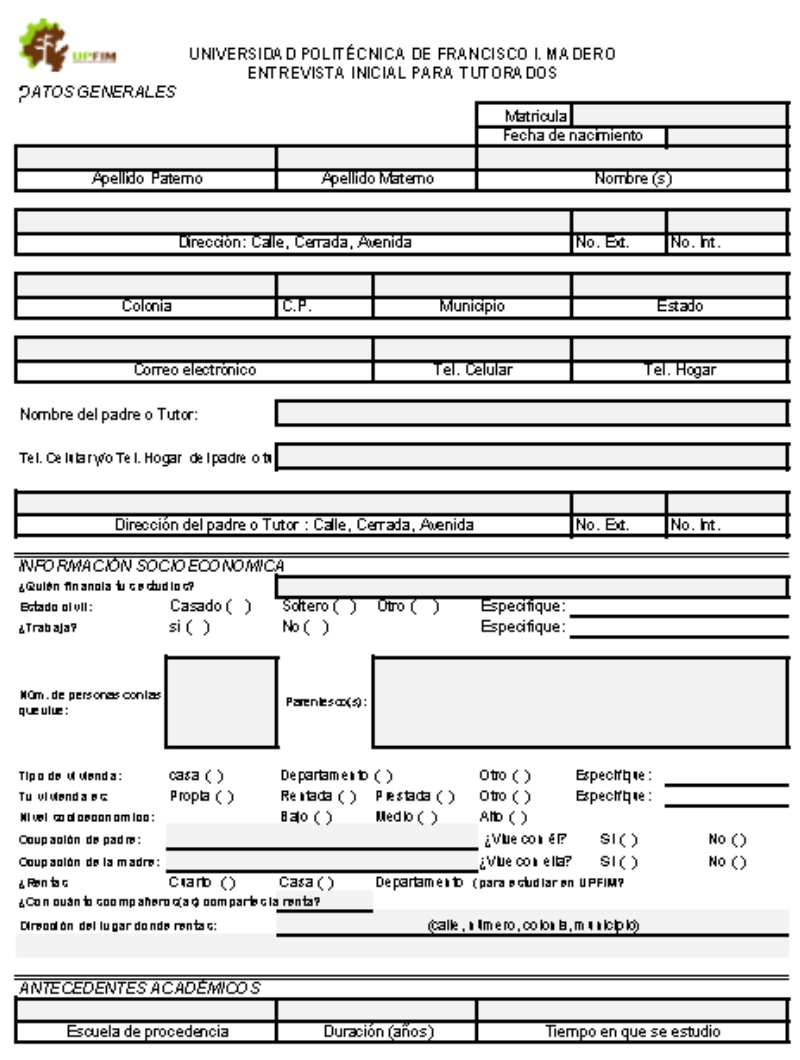

Figura 2 Entrevista inicial

Fuente: Elaboración Propia

2. Diseño. Usando los modelos EntidadRelación y Relacional de la base de datos se describe la estructura de la base de datos. Definiendo la forma en que la información se almacena y relaciona, tal como se presenta en las figuras 3 y 4 .

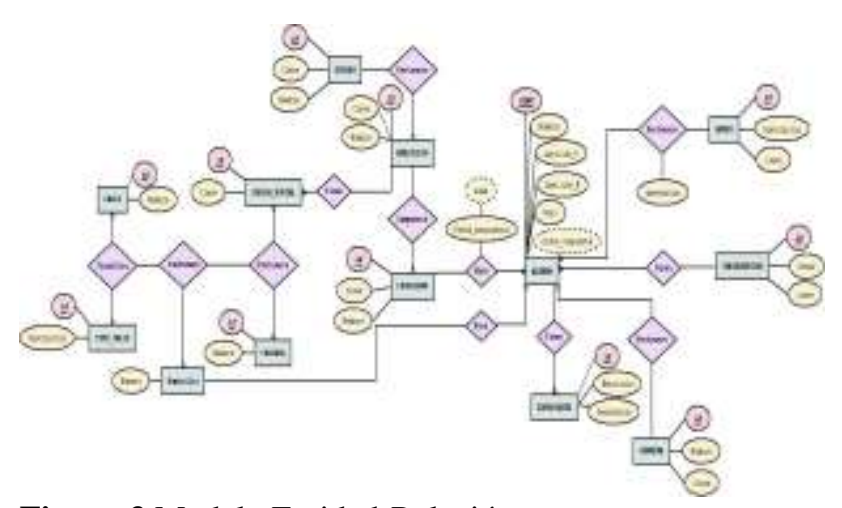

Figura 3 Modelo Entidad-Relación Fuente: Elaboración Propia

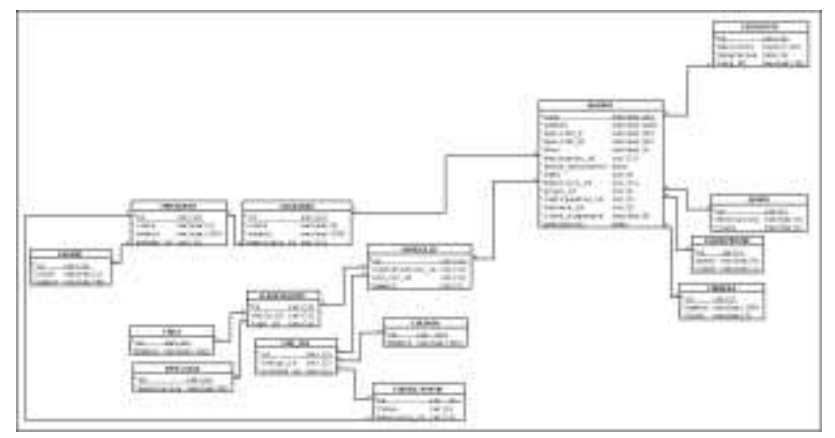

Figura 4 Modelo Relacional Fuente: Elaboración Propia

\section{Resultados y Conclusiones}

Una vez definida la estructura de la base de datos se realiza el diseño de la interfaz para el formulario, tal como se observa en las Figuras 5 y 6.

\section{Diseño de interfaz para el administrador de la base de datos}

Permite que el administrador pueda realizar la inserción de datos de manera práctica.

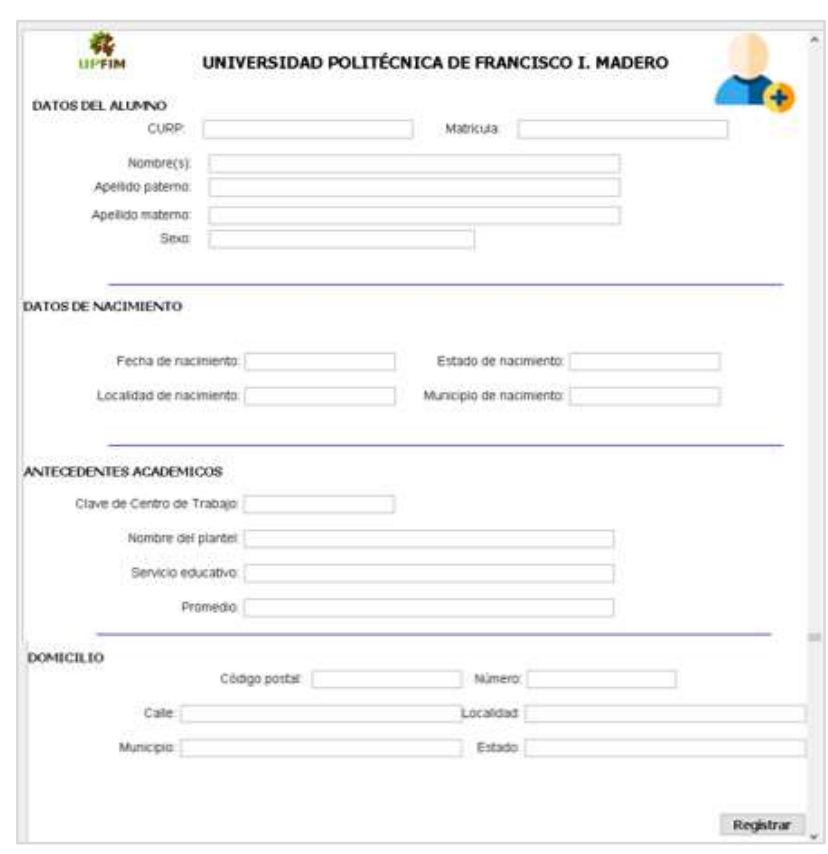

Figura 5 Interfaz para el administrador Fuente: Elaboración Propia

\section{Diseño de interfaz del formulario}

Es el medio mediante el cual el alumno contestará la encuesta de tutorías, como se realizara mediante la página del SICA de la Universidad, tiene que acoplarse al su diseño.

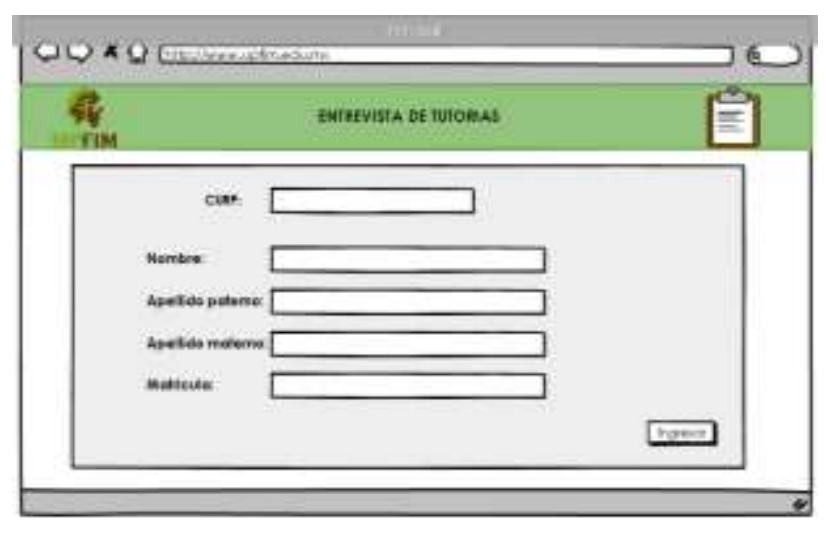

Figura 6 Página principal-Inicio Fuente: Elaboración Propia

HERNÁNDEZ-TAPIA, Zaila, REYNA-ÁNGELES, Omar, AVALOS-MARTÍNEZ, Giovanni y GÓMEZ-MENDOZA, Pablo Hernán. Diseño de una base de datos para la entrevista inicial de tutoría. Revista de Tecnología y Educación. 2020 


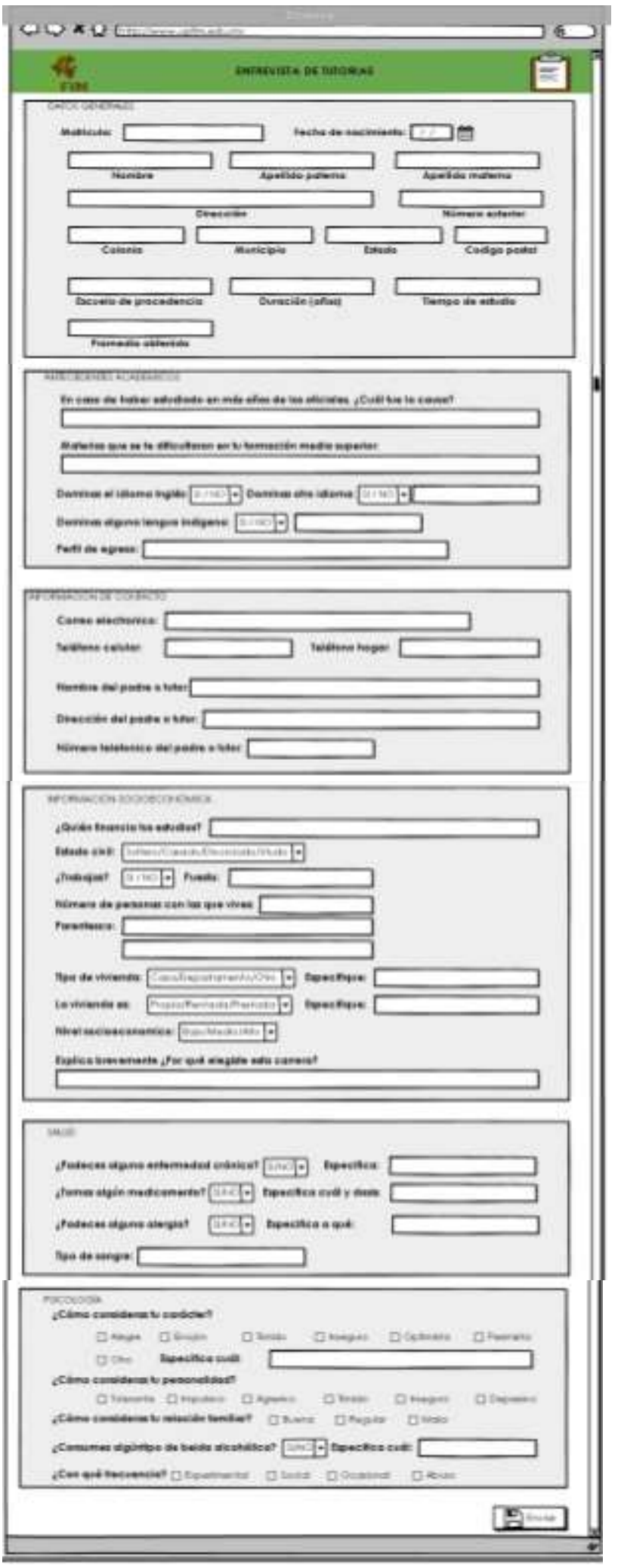

Figura 7 Entrevista

Fuente: Elaboración Propia

Para verificar que el diseño de la base de datos almacena los datos personales de los alumnos de forma: correcta, ordenada y validada; se realiza una prueba de escritorio de la estructura de la base de datos, tal como se presenta en la Figura 8.

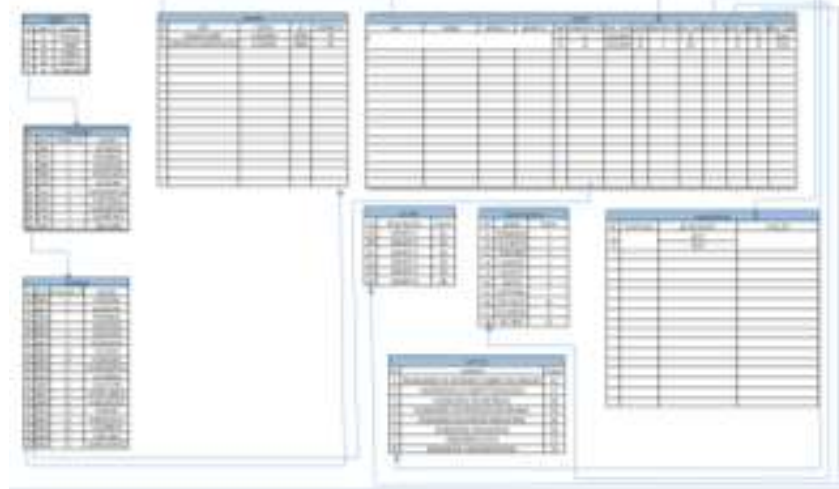

Figura 8 Prueba de escritorio

Fuente: Elaboración Propia

\section{Conclusiones}

En este trabajo se presentó el diseño de la base de datos y del formulario para la entrevista inicial de tutorías. Esto permitirá que cada tutor cuente, en forma digital, con información relevante y necesaria de cada uno de sus alumnos tutorados.

\section{Trabajos a futuro}

Se continuará con las siguientes etapas que son implementación, pruebas y mejoras, con la finalidad de verificar el funcionamiento del formulario y de ser necesario se realizarán las correcciones si es que presentara alguna falla o se implementaran mejoras.

\section{Referencias}

Abraham Silberschatz; Henry Korth; S. Sudarshan. (2014). Fundamentos de Bases de Datos. España: Mcgraw-Hill / Interamericana De España, S.A.

ANUIES, La educación superior en el siglo XXI, México, 1999, ANUIES.

ANUIES, Programa Institucional de tutorías, Serie Investigaciones, México, 2000, ANUIES.

ANUIES, Programas Institucionales de Tutoría. ANUIES. 2da. edición.

Ble, C. (s.f.). Modelo en cascada. (C. (. 20102013) Recuperado el 28 de marzo de 2016, http://librosweb.es/libro/tdd/capitulo_1/ modelo_en_cascada.html

Carrón, J. B. (2020). Desarrollo de un Sistema Web de Gestión de Tutorías Docentes. 
Marqués, Mercedes Universitat Jaume I. (2011). Bases de Datos: Castelló de la Plana.

Modelo del Sistema Integral de Tutorías. Subsistema de Universidades Politécnicas.

Molina Pozo, D. G. (2020). Herramientas web 2.0 para la enseñanza aprendizaje de la comunicación oral en Educación Inicial (Master's thesis, Quito).

Soriano Guasch, R. (2020). Ayuda al tutor: aplicación web para la gestión tutorial de las calificaciones en la educación secundaria.

Universidad Politécnica de Francisco I. Madero, Sistema Integral de Tutorías 2012.

Zaila Hernández \& Omar Reyna. (2015). Integración de áreas de Apoyo para la oportuna atención de la Acción Tutorial. En Proceedings T-III (33-41). México, S.C.: ECORFAN. 